\title{
Platform switching of implants may decrease bone loss
}

\section{Abstracted from}

\section{Chrcanovic BR, Albrektsson T, Wennerberg A.}

Platform switch and dental implants: A meta-analysis. J Dent 2015; 43: 629-646.

doi: 10.1016/j.jdent.2014.12.013. Epub 2015 Jan 2. Review. PubMed PMID: 25559693.

Address for correspondence: Bruno Chrcanovic, Department of Prosthodontics,

Faculty of Odontology, Malmö University, Malmö, Sweden. E-mail: bruno.chrcanovic@mah.se

\section{Question: Does platform switching of dental implants improve outcomes?}

Data sources PubMed/Medline, Web of Science and the Cochrane Oral Health Group Trials Register, clinicaltrials.gov, http://www. centerwatch.com/clinicaltrials, www. clinicalconnection.com supplemented by a manual search of dental implants-related journals. Study selection Clinical studies, either randomised or not, comparing implant failure rates, marginal bone level (MBL) and/or postoperative infection in any group of patients receiving platform-switched implants or platform-matched implants were considered.

Data extraction and synthesis Study quality was assessed using the Newcastle-Ottawa scale (NOS). Only randomised clinical trials (RCTs) were considered for meta-analysis. Implant failure and postoperative infection were the dichotomous outcomes measures evaluated. Weighted mean differences were used for MBL.

Results Twenty-eight studies (18 RCTs, six CCTs and four retrospective analyses) were included. Twenty-six studies were considered to be of high quality. Twelve hundred and sixteen platform-switched implants were included with 16 failures (1.32\%) and 1157 platform-matched implants and 13 failures (1.12\%). Twenty studies had no implant failures. In a meta-analysis for the outcome $\mathrm{MBL}$ (18-RCTs) there was less MBL loss at implants with platformswitching than at implants with platform-matching (mean difference $-0.29,95 \% \mathrm{Cl}-0.38$ to $-0.19 ; \mathrm{P}<0.00001$ )

Conclusions The results of the present study suggest that there is a significantly less MBL loss at implants with platform-switching than on implants with platform-matching. The results of the present review should be interpreted with caution due to the presence of uncontrolled confounding factors in the included studies, most of them with short follow-up periods.

\section{Commentary}

Introduced commercially in the late 1980s and early 1990s, wide diameter implants were often restored with standard diameter abutments because corresponding prosthetic components were frequently unavailable due to manufacturing production delays. ${ }^{1}$ Long term radiographic observation of these 'platform-switched', wide diameter implant restorations demonstrated a smaller than expected vertical change in the crestal bone height than typically observed with traditional matching components. ${ }^{2}$ This discovery provided the foundation for investigating the theories that attempt to explain this phenomenon as well as evaluating any outcomes associated with platform-switched versus platform-matched implants.

Coinciding with an increased level of interest by those in the scientific community, there has also been a general acceptance of the concept by practitioners and a commensurate growth in the number of clinical applications. Not surprisingly, this rise in demand has been followed by implant manufacturers releasing components designed to be mismatched, with the theory that these designs will aid in reducing initial bone loss and help to improve gingival contours and aesthetics. ${ }^{3}$

This comprehensive systematic review and meta-analysis of both prospective and retrospective clinical studies sought to compare three outcomes of platform-switched and platform-matched dental implants: the survival rates, postoperative infections and mean bone loss (MBL). Following the development of a well-focused PICO question, the authors conducted a structured systematic search of the published literature up to December 2014 looking for randomised and nonrandomised human studies: specifically those that compared the three outcomes of interest. Major strengths identified in their approach included the fact that they followed the PRISMA statement guidelines which is an evidence-based minimum set of items for reporting in systematic reviews and meta-analyses, ${ }^{4}$ and they employed recognised best practices (Newcastle-Ottawa Scale - NOS) for appraising the quality of any of the nonrandomised studies that were included in the eligible systematic reviews. ${ }^{5}$ Of the twenty-eight studies included in the qualitative synthesis, twentysix were deemed to be of high quality with two being considered moderate quality according to the Newcastle-Ottawa Scale (NOS). The authors elected to only consider randomised clinical trials (RCTs) for the meta-analysis (eighteen of which were included in this quantitative synthesis), and in an effort to gather as much pertinent data as possible, they contacted the authors of the studies included in the final analysis for possible missing data. 
To determine the risk of publication bias the authors chose a funnel plot looking for any asymmetry in the results, which may be indicative of bias. To their credit, the authors chose to use Standard Error for the y-axis when evaluating the outcome event of 'MBL' which is consistent with the recommended measure of study size. ${ }^{6}$ When analysing this outcome no clear asymmetry was evident, so it was deemed to indicate an absence of publication bias.

The authors were very clear in cautioning the reader when interpreting their results, as there were several inherent limitations within the meta-analysis. With so many confounding factors, it was difficult to draw strong conclusions, with much of the research in this area being limited by small cohort size and short follow-up periods. There was also a low level of specificity in several of the included studies where assessing platform-switched implants was not the primary focus of the investigation.

The stated purpose of this study was to test the null hypothesis of no difference in three outcomes for platform-switched versus platform-matched implants. While this well conducted systematic review and meta-analysis sheds further light on the subject of the benefits of platform-switched implants, it is disappointing that due to the lack of satisfactory data, meta-analyses for two of the three outcomes (implant failure and postoperative infection) could not be performed and therefore the authors could not estimate the influence that platform-switching has on these critical outcome measures. This meta-analysis also expressed cautious interpretation of their results partly due to short follow-up periods, but stated that the tendency favoured the platform-switch technique to prevent or minimise peri-implant marginal bone loss.

A previous summary review by this author asserted that even with the limited long-term data available at the time, the inward shift of the implant-abutment junction (IAJ) was a desirable morphological feature that may preserve crestal bone levels. ${ }^{7}$ Two recent systematic reviews and meta-analyses also compared crestal bone loss with platform-switched and platform-matched implants. One reached a similar conclusion to this meta-analysis in that the greater the mismatch between the implant platform and the abutment, the greater the bone preservation, ${ }^{8}$ while the other suggested that there was significantly less MBL with a platformswitched implant as compared to a platform-matched implant. ${ }^{9}$ These two studies corroborate the results from this systematic review and meta-analysis and also inform the reader that we are still awaiting longer-term clinical investigations whereby more definitive conclusions as to the theorised benefits of platformswitching may be drawn.

\section{Practice point}

- Patients expect their implant-supported restorations to maintain their aesthetic appearance over time, and the design of the implant can influence the factors necessary to establish and maintain this outcome.

- There is significantly less MBL with platform-switched implants than with platform-matched implants.

- There is an increase in the mean difference of MBL between the two approaches with an increase in follow-up time and with an increase in the mismatch between the implant platform and the abutment.

Gary L Stafford

Department of General Dental Sciences, Marquette University School of Dentistry Milwaukee, Wisconsin USA

1. Atieh MA, Ibrahim HM, Atieh AH. Platform switching for marginal bone preservation around dental implants: a systematic review and meta-analysis. J Periodontol 2010; 81: 1350-1366.

2. Lazzara RJ, Porter SS. Platform switching: a new concept in implant dentistry for controlling postrestorative crestal bone levels. Int J Periodontics Restorative Dent 2006; 26: 9-17.

3. Guerra F, Wagner W, Wiltfang I, et al. Platform switch versus platform match in the posterior mandible - 1 -year results of a multicenter randomized clinical trial. / Clin Periodontol 2014; 41: 521-529.

4. Moher D, Liberati A, Tetzlaff J, Altman DG; PRISMA Group. Preferred reporting items for systematic reviews and meta-analyses: the PRISMA statement. Ann Intern Med 2009; 151: 264-269.

5. Wells GA, Shea B, O'Connell D, Peterson J, Welch V, Losos M, Tugwell P. The Newcastle-Ottawa Scale (NOS) for assessing the quality of nonrandomized studies in meta-analyses. Available at: http://www.ohri.ca/programs/clinical_epidemiology/ oxford.asp [Accessed 17 August 2015].

6. Sterne JA, Egger M. Funnel plots for detecting bias in meta-analysis: guidelines on choice of axis. J Clin Epidemiol 2001; 54: 1046-1055.

7. Stafford GL. Evidence supporting platform-switching to preserve marginal bone levels not definitive. Evid Based Dent 2012; 13: 56-57.

8. Herekar M, Sethi M, Mulani S, Fernandes A, Kulkami H. Influence of platform switching on periimplant bone loss: a systematic review and meta-analysis. Implant Dent 2014; 23: 439-450.

9. Strietzel FP, Neumann K, Hertel M. Impact of platform switching on marginal periimplant bone-level changes. A systematic review and meta-analysis. Clin Oral Implants Res 2015; 26: 342-358.

Evidence-Based Dentistry (2015) 16, 84-85. doi:10.1038/sj.ebd.6401117 